\begin{tabular}{|c|c|}
\hline Title & Spatially-dependent nuclear reactor kinetic cal culations with the explicit fission product model \\
\hline Author(s) & Katagiri, Koji; Chiba, Go \\
\hline Citation & $\begin{array}{l}\text { A nnals of nuclear energy, 133, 202-208 } \\
\text { https://doi. org/10.1016j.anucene.2019.05.024 }\end{array}$ \\
\hline Issue Date & 2019-11 \\
\hline DOC URL & http:/hdl.handle.net/2115/83119 \\
\hline Rights & $\begin{array}{l}\text { (2) 2019. Ins manuscript version is made avallable under the CC-BY-IC-IVD } 4.0 \text { IIcense } \\
\text { http://reativecommons.org/icenses/by-nc-nd/4.0/ }\end{array}$ \\
\hline Rights(URL) & http:/creativecommons.org/icenses/by-nc-nd/4.0/ \\
\hline Type & article (author version) \\
\hline File Information & main.pdf \\
\hline
\end{tabular}

Instructions for use 


\title{
Spatially-dependent nuclear reactor kinetic calculations with the explicit fission product model
}

\author{
Koji Katagiria ${ }^{\mathrm{a}}$, Go Chiba*,a \\ ${ }^{a}$ Hokkaido University, Kita-ku, Sapporo, Hokkaido 060-8628, Japan
}

\begin{abstract}
In order to simulate complicated physical phenomena induced by fission product nuclides directly, we have developed the explicit fission product nuclides (EFP) model and have applied to one-point nuclear reactor kinetics problems in the past. In the present study, we extend the EFP model to spatially-dependent kinetics problems. Explicit representations of neutron diffusion kinetic equations with the EFP model are provided, and a new numerical method, a partial matrix exponential (PME) method, is proposed to solve differential equations for FP nuclides number densities analytically. Verification tests of the PME method against the $\theta$ method with fine time discretization are carried out, and the PME method is well verified. Numerical tests reveal that accuracy of the PME method is better than the $\theta$ method in coarse time mesh discretization, but accuracy of these two methods is comparable in typical time mesh discretization in actual kinetic calculations.
\end{abstract}

Also to demonstrate effectiveness and usefulness of the EFP model, we carry out several two-dimensional kinetics calculations with the EFP model considering leakage of gaseous FP nuclides from nuclear fuel. It is shown that gaseous FP leakage affects reactor power transient and this effect is dependent on magnitude of the leakage. Time transient of number densities of some FP nuclides is presented, and this shows different trend specific to each of FP nuclides.

Key words: nuclear reactor kinetics, fission product nuclides, matrix exponential method

\section{Introduction}

It is well known that delayed neutrons play quite important roles in nuclear fission reactors operations. In order to understand and accurately predict kinetic characteristics of nuclear fission reactors, behavior of fission product (FP) nuclides which can emit delayed neutrons, which are referred to as delayed neutron precursors, are as important as neutrons themselves.

*Corresponding author, Tel: +81-10-706-6683, Fax: +81-10-706-6683

Email address: go_chiba@eng.hokudai.ac.jp (Go Chiba) 
There exist a large number of delayed neutron precursors and their parent nuclides over 300. Since explicit treatment of them in numerical calculations of nuclear fission reactors kinetics has been considered unrealistic, the pseudo precursor model, which is well known as the six-group model, proposed by Keepin(Keepin, 1957) has been widely used. This pseudo precursor model has resulted in a great success, but current significant advancement in computer technology motivates us to treat all the fission product nuclides explicitly in nuclear reactors kinetics calculations because of several reasons. One of them is that some physical phenomena, such as delayed gamma-ray emissions and leakage of gaseous FP nuclides from nuclear fuel in an accidental condition, can be directly treated in numerical calculations. It is not impossible to treat these phenomena by the pseudo precursor model, but it would require complicated procedure to determine which nuclides belong to each of pseudo precursors. Furthermore, new knowledge acquired from fundamental studies and researches in the field of nuclear physics can be easily benefited to nuclear reactors applications if this explicit model is used.

Based on the above motivations, our research group has launched development of the explicit FP nuclides (EFP) model for nuclear reactors kinetic calculations, and has carried out several kinetics calculations in one-point problems with various conditions(Katagiri, 2017). In the present study, we extend the EFP model to spatially-dependent problems. A numerical method specific to spatially-dependent kinetics calculations with the EFP model will be proposed and verified. Also in order to demonstrate effectiveness and usefulness of the EFP model, we will carry out several two-dimensional kinetics calculations with the EFP model focusing on leakage of gaseous FP nuclides from nuclear fuel.

\section{Theory and numerical procedure}

For better understanding, kinetic equations of the EFP model with $N$ FP nuclides in one-point problems are presented below(Katagiri, 2017):

$$
\begin{aligned}
\frac{d n(t)}{d t} & =\frac{k_{p}-1}{l} n(t)+\sum_{i=1}^{N} \lambda_{i} C_{i}(t) \operatorname{Pn}_{i}, \\
\frac{d C_{i}(t)}{d t} & =\frac{k_{p}}{l} \frac{y_{i}}{\nu_{p}} n(t)-\lambda_{i} C_{i}(t)+\sum_{j=1}^{N} \lambda_{j} C_{j}(t) P_{j \rightarrow i}, \quad(i=1,2, \cdots, N),
\end{aligned}
$$

where $n(t)$ is neutron density at time $t, l$ is a prompt neutron life-time and $\nu_{p}$ is the average number of prompt neutrons emitted by a fission reaction. Parameters $C_{i}, \lambda_{i}, y_{i}$ and $\operatorname{Pn}_{i}$ are density, decay constant, independent fission yield and a neutron emission probability per decay of the $i$ th FP nuclide. In the present work, we use common fission yield data for all the fissile nuclides for simplicity. $P_{j \rightarrow i}$ is a decay branching ratio which is a probability that a nuclide $i$ is produced when a nuclide $j$ decays. $k_{p}$ is a prompt neutron multiplication factor defined as $k_{p}=k(1-\beta)$, where $k$ is a usual neutron multiplication factor and $\beta$ is a 
delayed neutron fraction defined as

$$
\beta=\frac{\nu_{d}}{\nu}=\frac{\sum_{i=1}^{N} \bar{y}_{i} \mathrm{Pn}_{i}}{\nu},
$$

where $\nu_{d}$ is the average number of delayed neutrons emitted by a fission reaction and $\bar{y}_{i}$ is cumulative fission yield of nuclide $i$.

This one-point equation with the EFP model is extended to spatially-dependent multi-group neutron diffusion kinetic equations as

$$
\begin{aligned}
\frac{1}{v_{g}} \frac{\partial \phi_{g}(\mathbf{r}, t)}{\partial t}= & \nabla D_{g}(\mathbf{r}, t) \cdot \nabla \phi_{g}(\mathbf{r}, t)-\Sigma_{r, g}(\mathbf{r}, t) \phi_{g}(\mathbf{r}, t)+\sum_{g^{\prime} \neq g} \Sigma_{s, g^{\prime} \rightarrow g}(\mathbf{r}, t) \phi_{g^{\prime}}(\mathbf{r}, t) \\
& +\chi_{p, g}(1-\beta) \sum_{g^{\prime}} \nu \Sigma_{f, g^{\prime}}(\mathbf{r}, t) \phi_{g^{\prime}}(\mathbf{r}, t)+\sum_{i=1}^{N} \chi_{d, i, g} \lambda_{i} C_{i}(\mathbf{r}, t) \operatorname{Pn}_{i}, \quad(g=1,2, \cdots G), \\
\frac{\partial C_{i}(\mathbf{r}, t)}{\partial t}= & y_{i} P(\mathbf{r}, t)-\lambda_{i} C_{i}(\mathbf{r}, t)+\sum_{j=1}^{N} \lambda_{j} C_{j}(\mathbf{r}, t) P_{j \rightarrow i}, \quad(i=1,2, \cdots, N),
\end{aligned}
$$

where $g$ is denoted to as an energy group, $G$ is the total number of energy groups, and $P(\mathbf{r}, t)$ is a fission reaction rate at a spatial position $\mathbf{r}$ and $t$ defined as $P(\mathbf{r}, t)=\sum_{g^{\prime}} \Sigma_{f, g^{\prime}}(\mathbf{r}, t) \phi_{g^{\prime}}(\mathbf{r}, t)$. Other notations related to the multi-group neutron diffusion equation are classical.

In the conventional pseudo precursor model, we do not have to consider FP nuclides (precursors) generations by decay of other FP nuclides, so number density of each FP nuclide is independent on each other. In such cases, each precursor density can be analytically calculated by assuming a change of neutron flux with time as the first(or second)-order polynomial function(Stacy, 1969), and this numerical procedure has been generally applied to many existing nuclear reactor kinetic calculation codes. On the other hand, in the EFP model, number density of each FP nuclide is dependent on each other, so this analytical procedure cannot be directly adopted to FP nuclides number density calculations. In Stacy's text(Stacy, 1969), it is mentioned that this analytical procedure and the familiar $\theta$-method give essentially identical results. This is because life-time of prompt neutrons is much shorter than those of pseudo delayed neutron precursors and time-dependence of prompt neutrons is relatively significant in kinetic calculations in the pseudo precursor model. On the other hand, in the EFP model, some short-lived FP nuclides are explicitly considered, so it is expected that more accurate procedure than the $\theta$-method is required. In the present paper, we propose an analytical procedure for FP nuclides number density calculations with the EFP model which is consistent with one for the pseudo precursor model. This procedure is described below.

After the spatial discretization, the spatially-dependent kinetic equations with the EFP model can be written in the following matrix form:

$$
\frac{d \mathbf{n}}{d t}=\mathbf{A n}(t)
$$


where $\mathbf{n}^{T}=\left(\mathbf{n}_{1}^{T}, \mathbf{n}_{2}^{T}, \cdots, \mathbf{n}_{M}^{T}\right)$ and $M$ is the total number of spatial meshes. The superscript of $T$ is used for matrix and vector transpositions. The vector $\mathbf{n}_{i}$ contains neutron flux and FP number density data of the $i$ th spatial mesh, and is defined as $\mathbf{n}_{i}^{T}=\left(\boldsymbol{\phi}_{i}^{T}, \mathbf{C}_{i}^{T}\right)$, where $\phi_{i}=\left(\phi_{i, 1}, \cdots, \phi_{i, G}\right)^{T}$ and $\mathbf{C}_{i}=\left(C_{i, 1}, \cdots, C_{i, N}\right)^{T}$. Thus the matrix $\mathbf{A}$ can be decomposed into $M \times M$ submatrices, and each of these submatrices, $\mathbf{A}_{i j}$, can be further decomposed into $2 \times 2$ submatrices as

$$
\mathbf{A}_{i j}=\left(\begin{array}{cc}
\mathbf{A}_{i j}^{\phi, \phi} & \mathbf{A}_{i j}^{\phi, C} \\
\mathbf{A}_{i j}^{C, \phi} & \mathbf{A}_{i j}^{C, C}
\end{array}\right) .
$$

Note that $\mathbf{A}_{i j}^{\phi, C}, \mathbf{A}_{i j}^{C, \phi}$ and $\mathbf{A}_{i j}^{C, C}$ are zero matrices if $i \neq j$. When spatial transport of FP nuclides is considered, $\mathbf{A}_{i j}^{C, C}$ becomes non-zero even if $i \neq j$. However, this is not considered in the present study because instantaneous removal of specific FP nuclides from a considered system is assumed. It is well known that Eq. (6) can be solved by the matrix exponential method in one-group one-point problems: a case of $G=1$ and $M=1$. On the other hand, the matrix exponential method cannot be applied in spatiallydependent multi-group problems since the matrix exponential $\exp (\mathbf{A} t)$ cannot be accurately calculated with existing numerical methods. Here we propose a new method, a partial matrix exponential (PME) method. Let us consider the following differential equation for $\phi_{i}$ and $\mathbf{C}_{i}$ :

$$
\frac{d}{d t}\left(\begin{array}{c}
\phi_{i} \\
\mathbf{C}_{i}
\end{array}\right)=\left(\begin{array}{cc}
\mathbf{A}_{i i}^{\phi, \phi} & \mathbf{A}_{i i}^{\phi, C} \\
\mathbf{A}_{i i}^{C, \phi} & \mathbf{A}_{i i}^{C, C}
\end{array}\right)\left(\begin{array}{c}
\boldsymbol{\phi}_{i} \\
\mathbf{C}_{i}
\end{array}\right)+\left(\begin{array}{c}
\mathbf{b} \\
\mathbf{0}
\end{array}\right),
$$

where a vector $\mathbf{b}$ corresponds to contributions of neutron flux in other spatial meshes. We know that the matrix exponential $\exp \left(\mathbf{A}_{i i}^{C, C} t\right)$ can be accurately calculated with existing numerical methods, so we attempt to adopt the matrix exponential method partially to the above equation.

Now let us consider the following differential equation with a generalized notation:

$$
\frac{d}{d t}\left(\begin{array}{l}
\mathbf{x}_{1}(t) \\
\mathbf{x}_{2}(t)
\end{array}\right)=\left(\begin{array}{ll}
\mathbf{A}_{11} & \mathbf{A}_{12} \\
\mathbf{A}_{21} & \mathbf{A}_{22}
\end{array}\right)\left(\begin{array}{l}
\mathbf{x}_{1}(t) \\
\mathbf{x}_{2}(t)
\end{array}\right)+\left(\begin{array}{c}
\mathbf{b}(t) \\
\mathbf{0}
\end{array}\right) .
$$

We assume that the matrix exponential $\exp \left(\mathbf{A}_{22} t\right)$ can be accurately calculated and the size of a vector $\mathbf{x}$, $\mathbf{x}_{1}$ and $\mathbf{x}_{2}$ are $I, I_{1}$ and $I_{2}$, respectively. Note that $I=I_{1}+I_{2}$.

Equation (9) can be rewritten as

$$
\begin{aligned}
& \frac{d \mathbf{x}_{1}(t)}{d t}=\mathbf{A}_{11} \mathbf{x}_{1}(t)+\mathbf{A}_{12} \mathbf{x}_{2}(t)+\mathbf{b}(t), \\
& \frac{d \mathbf{x}_{2}(t)}{d t}=\mathbf{A}_{21} \mathbf{x}_{1}(t)+\mathbf{A}_{22} \mathbf{x}_{2}(t) .
\end{aligned}
$$

By multiplying $\exp \left(-\mathbf{A}_{22} t\right)$ to both the sides of Eq. (11) from the left, the following equation can be derived since $\exp \left(-\mathbf{A}_{22} t\right) \mathbf{A}_{22}=\mathbf{A}_{22} \exp \left(-\mathbf{A}_{22} t\right)$ :

$$
\frac{d}{d t}\left(\exp \left(-\mathbf{A}_{22} t\right) \mathbf{x}_{2}(t)\right)=\exp \left(-\mathbf{A}_{22} t\right) \mathbf{A}_{21} \mathbf{x}_{1}(t)
$$


By integrating Eq. (12) over $[t, t+\Delta t]$, the following equation can be derived:

$$
\exp \left(-\mathbf{A}_{22}(t+\Delta t)\right) \mathbf{x}_{2}(t+\Delta t)-\exp \left(-\mathbf{A}_{22} t\right) \mathbf{x}_{2}(t)=\int_{t}^{t+\Delta t} \exp \left(-\mathbf{A}_{22} t^{\prime}\right) \mathbf{A}_{21} \mathbf{x}_{1}\left(t^{\prime}\right) d t^{\prime} .
$$

By multiplying $\exp \left(\mathbf{A}_{22}(t+\Delta t)\right)$ to this equation from the left, the following equation can be derived since $\exp (\mathbf{A}) \exp (\mathbf{B})=\exp (\mathbf{A}+\mathbf{B})$ when matrices of $\mathbf{A}$ and $\mathbf{B}$ are commuting matrices; $\mathbf{A B}=\mathbf{B} \mathbf{A}$ :

$$
\mathbf{x}_{2}(t+\Delta t)=\exp \left(\mathbf{A}_{22} \Delta t\right) \mathbf{x}_{2}(t)+\int_{t}^{t+\Delta t} \exp \left(\mathbf{A}_{22}\left(t-t^{\prime}+\Delta t\right)\right) \mathbf{A}_{21} \mathbf{x}_{1}\left(t^{\prime}\right) d t^{\prime} .
$$

If we introduce $\hat{t}=t^{\prime}-t$, an integration of the second term of the right hand side of Eq. (14) can be presented as

$$
\int_{0}^{\Delta t} \exp \left(\mathbf{A}_{22}(\Delta t-\hat{t})\right) \mathbf{A}_{21} \mathbf{x}_{1}(\hat{t}) d \hat{t}
$$

Here we introduce the following linear assumption to $\mathbf{x}_{1}(\hat{t})$ :

$$
\mathbf{x}_{1}(\hat{t})=\mathbf{x}_{1}(t)+\left(\mathbf{x}_{1}(t+\Delta t)-\mathbf{x}_{1}(t)\right) \frac{\hat{t}}{\Delta t} .
$$

By substituting this equation into Eq. (15), the integration of the second term of the right hand side of Eq. (14) can be rewritten as

$$
\exp \left(\mathbf{A}_{22} \Delta t\right)\left[\left(\int_{0}^{\Delta t} \exp \left(-\mathbf{A}_{22} \hat{t}\right) d \hat{t}\right) \mathbf{A}_{21} \mathbf{x}_{1}(t)+\frac{1}{\Delta t}\left(\int_{0}^{\Delta t} \hat{t} \exp \left(-\mathbf{A}_{22} \hat{t}\right) d \hat{t}\right)\left\{\mathbf{A}_{21}\left(\mathbf{x}_{1}(t+\Delta t)-\mathbf{x}_{1}(t)\right)\right\}\right] .
$$

Integrals in Eq. (17) can be calculated as

$$
\begin{aligned}
& \int_{0}^{\Delta t} \exp \left(-\mathbf{A}_{22} \hat{t}\right) d \hat{t}=-\mathbf{A}_{22}^{-1}\left(\exp \left(-\mathbf{A}_{22} \Delta t\right)-\mathbf{I}\right), \\
& \int_{0}^{\Delta t} \hat{t} \exp \left(-\mathbf{A}_{22} \hat{t}\right) d \hat{t}=-\mathbf{A}_{22}^{-1} \Delta t \exp \left(-\mathbf{A}_{22} \Delta t\right)-\left(\mathbf{A}_{22}^{-1}\right)^{2}\left(\exp \left(-\mathbf{A}_{22} \Delta t\right)-\mathbf{I}\right),
\end{aligned}
$$

where $\mathbf{I}$ is a $I_{2} \times I_{2}$ unit matrix. Thus Eq. (17) can be written as

$$
\begin{aligned}
\exp \left(\mathbf{A}_{22} \Delta t\right)\left[-\mathbf{A}_{22}^{-1}\left(\exp \left(-\mathbf{A}_{22} \Delta t\right)-\mathbf{I}\right) \mathbf{A}_{21} \mathbf{x}_{1}(t)\right. & \\
+ & \left.\left\{-\mathbf{A}_{22}^{-1} \exp \left(-\mathbf{A}_{22} \Delta t\right)-\frac{\left(\mathbf{A}_{22}^{-1}\right)^{2}}{\Delta t}\left(\exp \left(-\mathbf{A}_{22} \Delta t\right)-\mathbf{I}\right)\right\} \mathbf{A}_{21}\left(\mathbf{x}_{1}(t+\Delta t)-\mathbf{x}_{1}(t)\right)\right] \\
=\mathbf{A}_{22}^{-1}\left\{\exp \left(\mathbf{A}_{22} \Delta t\right)+\frac{\mathbf{A}_{22}^{-1}}{\Delta t}\left(\mathbf{I}-\exp \left(\mathbf{A}_{22} \Delta t\right)\right)\right\} & \mathbf{A}_{21} \mathbf{x}_{1}(t) \\
& +\mathbf{A}_{22}^{-1}\left\{-\mathbf{I}-\frac{\mathbf{A}_{22}^{-1}}{\Delta t}\left(\mathbf{I}-\exp \left(\mathbf{A}_{22} \Delta t\right)\right)\right\} \mathbf{A}_{21} \mathbf{x}_{1}(t+\Delta t)
\end{aligned}
$$

Here some variables are defined as follows:

87

88

$$
\begin{aligned}
& \mathbf{M}=\exp \left(\mathbf{A}_{22} \Delta t\right), \\
& \mathbf{E}=\mathbf{A}_{22}^{-1}\left\{\exp \left(\mathbf{A}_{22} \Delta t\right)+\frac{\mathbf{A}_{22}^{-1}}{\Delta t}\left(\mathbf{I}-\exp \left(\mathbf{A}_{22} \Delta t\right)\right)\right\} \mathbf{A}_{21}, \\
& \mathbf{X}=\mathbf{A}_{22}^{-1}\left\{-\mathbf{I}-\frac{\mathbf{A}_{22}^{-1}}{\Delta t}\left(\mathbf{I}-\exp \left(\mathbf{A}_{22} \Delta t\right)\right)\right\} \mathbf{A}_{21} . \\
& 5
\end{aligned}
$$


Then Eq. (14) can be written as follows:

$$
\mathbf{x}_{2}(t+\Delta t)=\mathbf{M} \mathbf{x}_{2}(t)+\mathbf{E} \mathbf{x}_{1}(t)+\mathbf{X} \mathbf{x}_{1}(t+\Delta t)
$$

After Eq. (10) is discretized over $[t, t+\Delta t]$ with known vectors $\mathbf{x}_{1}(t)$ and $\mathbf{x}_{2}(t)$, we have to consider two unknown vectors $\mathbf{x}_{1}(t+\Delta t)$ and $\mathbf{x}_{2}(t+\Delta t)$. Using Eq. (25), $\mathbf{x}_{2}(t+\Delta t)$ can be replaced by $\mathbf{x}_{1}(t+\Delta t)$ and the known parameters, so we can numerically calculate $\mathbf{x}_{1}(t+\Delta t)$, and then $\mathbf{x}_{2}(t+\Delta t)$ can be calculated with Eq. (25).

When we consider the spatially-dependent kinetic diffusion equations with the EFP model, a matrix $\mathbf{A}_{21}$ is replaced by $\mathbf{A}^{\phi, C}$ and we know that this matrix $\mathbf{A}^{\phi, C}$ is represented as $\mathbf{A}^{\phi, C}=\mathbf{y f}^{T}$ where $\mathbf{y}=\left(y_{1}, y_{2}, \cdots, y_{N}\right)^{T}$ and $\mathbf{f}=\left(\Sigma_{f, 1}, \Sigma_{f, 2}, \cdots, \Sigma_{f, G}\right)^{T}$. The vector $\mathbf{y}$ represents fission product nuclides generation by a fission reaction. A fission reaction rate at the $i$ th mesh can be presented as $P_{i}=\mathbf{f}^{T} \boldsymbol{\phi}_{i}$, so the matrices $\mathbf{M}, \mathbf{E}$ and $\mathbf{X}$ are replaced by the following matrix and vectors in the spatially-dependent kinetic problems if we assume that fission reaction rate during this time interval changes linearly:

$$
\begin{aligned}
\boldsymbol{\mu} & =\exp \left(\mathbf{A}^{C, C} \Delta t\right) \\
\boldsymbol{\eta} & =\left(\mathbf{A}^{C, C}\right)^{-1}\left\{\exp \left(\mathbf{A}^{C, C} \Delta t\right)+\frac{\left(\mathbf{A}^{C, C}\right)^{-1}}{\Delta t}\left(\mathbf{I}-\exp \left(\mathbf{A}^{C, C} \Delta t\right)\right)\right\} \mathbf{y}, \\
\boldsymbol{\xi} & =\left(\mathbf{A}^{C, C}\right)^{-1}\left\{-\mathbf{I}-\frac{\left(\mathbf{A}^{C, C}\right)^{-1}}{\Delta t}\left(\mathbf{I}-\exp \left(\mathbf{A}^{C, C} \Delta t\right)\right)\right\} \mathbf{y} .
\end{aligned}
$$

Note that $\boldsymbol{\mu}$ is a $N \times N$ matrix, and $\boldsymbol{\eta}$ and $\boldsymbol{\xi}$ are vectors whose sizes are $N$.

The above procedure results in the following time-discretized spatially-dependent kinetic diffusion equations with the EFP model corresponding to Eq. (10):

$$
\begin{array}{r}
-\nabla D_{g}(\mathbf{r}, t+\Delta t) \cdot \nabla \phi_{g}(\mathbf{r}, t+\Delta t)+\left(\Sigma_{r, g}(\mathbf{r}, t+\Delta t)+\frac{1}{\theta v_{g} \Delta t}\right) \phi_{g}(\mathbf{r}, t+\Delta t) \\
=\sum_{g^{\prime} \neq g} \Sigma_{s, g^{\prime} \rightarrow g}(\mathbf{r}, t+\Delta t) \phi_{g^{\prime}}(\mathbf{r}, t+\Delta t)+\chi_{p, g}(1-\beta) \sum_{g^{\prime}} \nu \Sigma_{f, g^{\prime}}(\mathbf{r}, t+\Delta t) \phi_{g^{\prime}}(\mathbf{r}, t+\Delta t) \\
+\sum_{i=1}^{N} \chi_{d, i, g} \lambda_{i} \operatorname{Pn}_{i} \xi_{i} P(\mathbf{r}, t+\Delta t)+Q_{e x}(\mathbf{r}, t)
\end{array}
$$

where

$$
Q_{e x}(\mathbf{r}, t)=\sum_{i=1}^{N} \chi_{d, i, g} \lambda_{i} \operatorname{Pn}_{i}\left\{(\boldsymbol{\mu} \mathbf{C}(\mathbf{r}, t))_{i}+\eta_{i} P(\mathbf{r}, t)\right\}+\frac{1-\theta}{\theta} R_{g}(\mathbf{r}, t)+\frac{\phi_{g}(\mathbf{r}, t)}{\theta v_{g} \Delta t}
$$

and

$$
R_{g}(\mathbf{r}, t)=\frac{\phi_{g}(\mathbf{r}, t)}{\theta v_{g} \Delta t}+\sum_{i=1}^{N} \chi_{d, i, g} \lambda_{i} \operatorname{Pn}_{i}\left\{C_{i}(\mathbf{r}, t)-\xi_{i} P(\mathbf{r}, t)\right\}-Q_{e x}(\mathbf{r}, t-\Delta t) .
$$

Note that the following $\theta$ method is introduced to discretize the time-dependent neutron diffusion equation in the above procedure:

$$
\int_{t}^{t+\Delta t} P\left(\mathbf{r}, t^{\prime}\right) d t^{\prime}=\{(1-\theta) P(\mathbf{r}, t)+\theta P(\mathbf{r}, t+\Delta t)\} \Delta t
$$


Equation (10) can be solved also with the $\theta$ method without any matrix exponential calculations. With the $\theta$ method, $\mathbf{x}_{2}(t+\Delta t)$ is calculated as follows:

$$
\mathbf{x}_{2}(t+\Delta t)=\mathbf{M}_{\theta} \mathbf{x}_{2}(t)+\mathbf{E}_{\theta} \mathbf{x}_{1}(t)+\mathbf{X}_{\theta} \mathbf{x}_{1}(t+\Delta t),
$$

where

$$
\begin{aligned}
\mathbf{M}_{\theta} & =\left(\mathbf{I}-\theta \Delta t \mathbf{A}_{22}\right)^{-1}\left(\mathbf{I}+(1-\theta) \Delta t \mathbf{A}_{22}\right) \\
\mathbf{E}_{\theta} & =(1-\theta) \Delta t\left(\mathbf{I}-\theta \Delta t \mathbf{A}_{22}\right)^{-1} \mathbf{A}_{21} \\
\mathbf{X}_{\theta} & =\theta \Delta t\left(\mathbf{I}-\theta \Delta t \mathbf{A}_{22}\right)^{-1} \mathbf{A}_{21}
\end{aligned}
$$

In the following numerical testing, this $\theta$ method is used to obtain reference solutions and to compare with the PME method.

\section{Numerical tests and results}

\subsection{Numerical conditions}

As already described, we use common fission yield data for all the fissile nuclides for simplicity in the present study. We choose fission yield data of uranium-235 induced by thermal neutrons provided in the JENDL fission product yield data file-2011 (JENDL/FPY-2011)(Katakura, 2011). Thus, only macroscopic cross sections can be used to define numerical problems. Regarding the decay data, the JENDL fission product decay data file-2011 (JENDL/FPD-2011)(Katakura, 2011) is used.

As shown in Eqs. (27) and (28), our PME method requires a matrix inversion calculation of $\mathbf{A}^{C, C}$, which defines nuclides decay and transmutation processes. Matrix inversion calculations of $\mathbf{A}^{C, C}$ cannot be done or give poor results if stable or long-lived nuclides are included in the matrix $\mathbf{A}^{C, C}$. In the present work, nuclides which do not contribute to delayed neutron emissions are neglected, so totally 401 nuclides are treated in the EFP model. In other words, nuclides neglected in this calculation and their daughter nuclides do not emit any delayed neutrons. All the stable and long-lived nuclides which deteriorate the above matrix inversion calculations are excluded. The number of delayed neutrons emitted by a fission reaction, $\nu_{d}$, is calculated based on the adopted fission yield and decay data.

Exponential matrix is numerically calculated by the 14th-order Chebyshev rational approximation method(Pusa, 2011). It is possible to use other methods such as the Páde approximation method and the mini-max polynomial approximation method(Kawamoto, 2015), but it is confirmed that any significant differences are not observed in results among these methods.

All the calculations are carried out with an in-house multi-dimensional diffusion kinetics code based on finite-volume spatial discretization. 


\subsection{Verification test of the in-house kinetics code for a two-dimensional TWIGL benchmark problem}

In this section, our in-house diffusion kinetics code is verified with a well-known two-dimensional TWIGL seed-blanket reactor kinetics benchmark. Core geometrical specification is shown in Fig. 1. Cross sections are defined in two-group as shown in Table $\mathbf{1}$ and the number of delayed neutron precursor families is 1. Delayed neutron fraction $\beta$ is set 0.0075 , and group-wise neutron speeds $v_{1}$ and $v_{2}$ are $1.0 \times 10^{7} \mathrm{~cm} / \mathrm{s}$ and $2.0 \times 10^{5} \mathrm{~cm} / \mathrm{s}$. In the present work, a step perturbation case is considered; a stationary critical state is considered as an initial condition and then an absorption cross section of group 2 in the material 1 is changed as $\Sigma_{a, 2}(t)=\Sigma_{a, 2}(0)-0.0035$ in $t>0$. To make this system critical as a initial condition, an eigenvalue equation is numerically solved to obtain $k_{\text {eff }}$, and $\nu \Sigma_{f}$ is divided by this $k_{\text {eff }}$. Reference solutions of this benchmark problem have been provided by Sutton et al.(Sutton, 1996), and it has been widely utilized for verification calculations of newly-developed kinetics codes.(Ban, 2011) In our verification calculations, the number of spatial meshes is the same as that in the reference calculation, and the time discretization is carried out with the $\theta$ method with $\theta=0.5$. Reactor power at several time points after the perturbation is shown in Table 2. Good agreement is observed, so our in-house diffusion kinetics code has been well verified.

\subsection{Verification and performance test of the PME method in a one-dimensional problem}

In order to verify the PME method, kinetic calculations are carried out in a one-dimensional problem. This problem is a one-dimensional slab core simulating a thermal neutron system, in which a fuel slab with $60 \mathrm{~cm}$ thickness is sandwiched by reflector slabs with $30 \mathrm{~cm}$ thickness. One spatial mesh per $1 \mathrm{~cm}$ is assigned. Cross sections are defined in two-group as shown in Table 3. Delayed neutron fraction $\beta$ is set 0.0075 , and the total number of neutrons $\nu$ is determined from this $\beta$ and $\nu_{d}$. Group-wise neutron speeds $v_{1}$ and $v_{2}$ are $1.0 \times 10^{7} \mathrm{~cm} / \mathrm{s}$ and $2.0 \times 10^{5} \mathrm{~cm} / \mathrm{s}$. Neutron generation time of this system is calculated around $6 \times 10^{-5} \mathrm{~s}$ from forward and adjoint neutron fluxes of the eigenvalue equations.

Here we consider a transient after step-wise positive reactivity insertion to a stationary critical state. To make this system critical, an eigenvalue equation is numerically solved to obtain $k_{\text {eff }}$, and $\nu \Sigma_{f}$ is divided by this $k_{\text {eff }}$. Positive reactivity is inserted by reducing $\Sigma_{a, 2}$ in a fuel region 0.00025 . This corresponds to a reactivity insertion of around $+0.001 \Delta k / k k^{\prime}$. Time profiles of reactor power and FP number densities during one second after the reactivity insertion are concerned. Reference solution is obtained by the $\theta$ method with fine time discretization: $\Delta t=0.0001 \mathrm{~s}$.

Accuracy of the PME method is quantified as follows. Reactor power and FP nuclides number densities at ten time points $(t=0.1,0.2, \cdots, 1.0)$ after the reactivity insertion are calculated, and relative differences to references are obtained at these time points. After that, maximum and root-mean-square (RMS) values of absolute values of these relative differences are calculated. 
Maximum and RMS errors of the PME method with different time mesh sizes for reactor power are shown in Fig. 2. Also, maximum and RMS errors of the PME method for FP number densities are shown in Fig. 3. Smaller errors are obtained in both reactor power and FP nuclides densities when finer time mesh is adopted. These results clearly show that the PME method works properly.

Next let us discuss the accuracy of the PME method and the $\theta$ method. Reactor power transients calculated by these two methods with $\Delta t=0.1 \mathrm{~s}$ are shown in Fig. 4 . Because of the coarse time discretization, both the methods provide oscillating results compared with the reference, and its magnitude is almost identical between these two methods. On the other hand, in some FP number densities, non-negligible differences are observed between these two methods. As an example, transients of number density of cobalt-78, whose half-life is $4.84 \mathrm{~ms}$, are shown also in Fig. 4. Both the two methods give oscillating results, but its magnitude of the PME method is smaller than that of the $\theta$ method. RMS errors of FP number densities of the PME method and the $\theta$ method are shown in Fig. 5. When coarse time discretization, $\Delta t=0.1$, is adopted, more accurate results can be obtained with the PME method than with the $\theta$ method. On the other hand, when finer time discretization is adopted, these two methods give almost identical results. Note that the $\theta$ method gives slightly better results in some nuclides, but this reason has not yet been investigated. These trends observed in the RMS errors are also found in the maximum errors of FP number densities. From the above results, it can be concluded that there are not significant differences between these two methods in typical time mesh discretization in actual kinetics calculations.

\subsection{A two-dimensional problem with leakage of gaseous fission product nuclides}

As mentioned above, the EFP model is suitable to kinetics calculations with gaseous FP nuclides leakage. In the present study, leakage of gaseous FP nuclides from fuel is considered with the following simple model; it is assumed that certain ratio of the FP nuclides is instantaneously removed from a system. To do that, a leakage constant (LC), $\lambda_{L}$, is defined. During a short time period $\Delta t$, a fraction $\lambda_{L} \Delta t$ of each FP nuclide instantaneously disappear. To take into account this in actual calculations, we need to modify the diagonal element of the matrix $\mathbf{A}^{C, C}$. If a leakage constant and a decay constant of a FP nuclide are $\lambda_{L}$ and $\lambda$, a diagonal element corresponding to this nuclide in $\mathbf{A}^{C, C}$ becomes $-\left(\lambda+\lambda_{L}\right)$. Other elements in this matrix related to the decay of this nuclide should be unchanged. Rigorously speaking, leakage of gaseous FP nuclides also affects macroscopic absorption cross sections, but absorption reaction rate of these nuclides are negligibly small in comparison with leakage in concerning time range of the present study $\left(\lambda_{L}>0.01\right)$. When slow and long-period transient due to the gaseous FP leakage is considered, leakage effect on neutron absorption should be taken into account.

Kinetics calculations with leakage consideration of gaseous FP nuclides are carried out with the twodimensional TWIGL core utilized in the preceding verification calculations. In this calculation, all isotopes of krypton, xenon and iodine are regarded as gaseous FP nuclides. All the calculations are performed with 
the PME method with $\Delta t=0.01 \mathrm{~s}$.

First we consider gaseous FP leakage in a stationary critical state. All gaseous FP nuclides in a core begin to leak from a system with common values of leakage coefficients at $t=0$. Here we concern reactor power and number densities of the following three nuclides: bromine-87 (halflife is $55.7 \mathrm{~s}$ ), cobalt-78 (4.84 ms) and iodine-137 (24.5 s). Figure 6 shows reactor power change with time. Reactor power begins to decrease at $t=0$ when gaseous FP leakage starts, and it becomes significant if the leakage constant becomes large. Number densities of Br-87, Co-78 and I-137 are shown in Fig. 7. Change of the Br-87 number density is slightly affected by the gaseous FP leakage because this is not a leaked nuclide and its half-life is relatively long. Change of the Co-78 number density is quite similar with reactor power change due to its short half-life. Change of the I-137 number density is significant because this nuclide is one of the gaseous FP nuclides.

Next we consider a step-wise positive reactivity insertion accompanied with gaseous FP nuclides leakage. In this case, the same perturbation in the verification calculation is introduced. Figure 8 shows reactor power change. Reactor power gradually increases after the prompt jump, but this increase is restrained by gaseous FP leakage. Number densities of Br-87, Co-78 and I-137 are shown in Fig. 9. The similar trends are observed like gaseous FP leakage in a critical state.

Note that purpose of the above calculations is to demonstorate usefulness of our EFP model in spatiallydependent kinetics problems, and quite a simplified model about leakage of gaseous fission product nuclides is adopted. When one attempts to adopt the EFP model to actual gaseous FP leakage problems, one has to consider mechanical and chemical behavior of gaseous FP nuclides such as xenon and iodine in a nuclear fuel pellet and a gap between a pellet and cladding.

\section{Conclusion}

In order to simulate complicated physical phenomena induced by fission product nuclides directly, we have developed the EFP model and have applied to one-point nuclear reactor kinetics problems in the past. In the present study, we have extended the EFP model to spatially-dependent kinetics problems. Explicit representations of neutron diffusion kinetic equations with the EFP model have been provided, and a new numerical method, the PME method, has been proposed to solve differential equations for FP nuclides number densities analytically. Verification tests of the PME method against the familiar $\theta$ method with fine time discretization have been carried out, and the PME method has been well verified. Numerical tests have revealed that accuracy of the PME method is better than the $\theta$ method in coarse time mesh discretization, but accuracy of these two methods is comparable in typical time mesh discretization in actual kinetic calculations.

Also to demonstrate effectiveness and usefulness of the EFP model, we have carried out several two- 
dimensional kinetics calculations with the EFP model considering leakage of gaseous FP nuclides from nuclear fuel. It has been shown that gaseous FP leakage affects reactor power transient and this effect is dependent on magnitude of the leakage. Time transient of number densities of some FP nuclides has been presented, and this shows different trend specific to each of FP nuclides.

As a future subject, we have a plan to apply the present EFP model to transient in which a system is over prompt critical. It is expected that we can simulate easily transport and energy deposition of delayed gamma-ray which give negative feedback in this transient. Improvement of gaseous FP leakage simulations, such as local leakage problems and transport of leaked FP nuclides rather than instantaneous escape from a system, is also a future subject.

\section{Acknowledgements}

This work is motivated by a unpublished work about modeling of volatile delayed neutron precursors in disruptive fast reactor core by Dr. Toshihisa Yamamoto of nuclear regulation authority of Japan. This work was supported by JSPS KAKENHI Grant Number JP 16 K18344.

\section{References}

Ban, Y., Endo, T., Yamamoto, A., Yamane, Y., 2011. Explicit time integration scheme using Krylov subspace method for reactor kinetics equation, J. Nucl. Sci. Technol., 48, 243-255.

Katagiri, K., Chiba, G., 2017. Nuclear reactor one-point kinetic calculations with explicit fission product model, Proc. of Reactor Physics Asia 2017, RPHA2017, Chengdu, China, Aug. 24-25, 2017. [CD-ROM]

Katakura, J., 2011. JENDL FP decay data file 2011 and fission yields data file 2011, JAEA-Data/Code 2011-025, Japan Atomic Energy Agency.

Kawamoto, Y., Chiba, G., Tsuji, M., Narabayashi, T., 2015. Numerical solution of matrix exponential in burn-up equation using mini-max polynomial approximation, Ann. Nucl. Energy, 80, 219-224.

Keepin, G.R., et al., 1957. Delayed neutrons from fissionable isotopes of uranium, plutonium and thorium, Phys. Rev., 107, 1044-1049

Pusa, M., 2011. Approximation to the matrix exponential in burnup calculations, Nucl. Sci. Eng., 169, 155-167.

Sutton, T.M., Aviles, B.N., 1996. Diffusion theory methods for spatial kinetics calculation, Prog. Nucl. Energy, 30, 119-182.

Stacy, W.M., 1969. Space-time nuclear reactor kinetics, Academic press, New York. 
Table 1: Two-group cross sections in a two-dimensional TWIGL core

\begin{tabular}{ccccccc}
\hline Material & $g$ & $D_{g}[\mathrm{~cm}]$ & $\Sigma_{a, g}[/ \mathrm{cm}]$ & $\nu \Sigma_{f, g}[/ \mathrm{cm}]$ & $\chi_{g}$ & $\Sigma_{g \rightarrow 2}[/ \mathrm{cm}]$ \\
\hline \multirow{2}{*}{$1 / 2$} & 1 & 1.4 & 0.010 & 0.007 & 1.0 & 0.01 \\
& 2 & 0.4 & 0.150 & 0.200 & 0.0 & 0.0 \\
\hline 3 & 1 & 1.3 & 0.008 & 0.003 & 1.0 & 0.01 \\
& 2 & 0.5 & 0.050 & 0.060 & 0.0 & 0.0 \\
\hline
\end{tabular}


Table 2: Core relative power in the TWIGL benchmark with step perturbation

\begin{tabular}{cccc}
\hline Time $[\mathrm{s}]$ & Reference & \multicolumn{2}{c}{ Present work } \\
& $(\Delta t=0.0001 \mathrm{~s})$ & $(\Delta t=0.01 \mathrm{~s})$ & $(\Delta t=0.001 \mathrm{~s})$ \\
\hline 0.0 & 1.000 & 1.000 & 1.000 \\
0.1 & 2.062 & 2.061 & 2.062 \\
0.2 & 2.079 & 2.078 & 2.079 \\
0.3 & 2.096 & 2.096 & 2.096 \\
0.4 & 2.114 & 2.113 & 2.114 \\
0.5 & 2.131 & 2.131 & 2.132 \\
\hline
\end{tabular}


Table 3: Two-group cross sections in a one-dimensional slab core

\begin{tabular}{ccccccc}
\hline Material & $g$ & $D_{g}[\mathrm{~cm}]$ & $\Sigma_{a, g}[/ \mathrm{cm}]$ & $\nu \Sigma_{f, g}[/ \mathrm{cm}]$ & $\chi_{g}$ & $\Sigma_{g \rightarrow 2}[/ \mathrm{cm}]$ \\
\hline Fuel & 1 & 1.58 & 0.0032 & 0.0 & 1.0 & 0.0178 \\
& 2 & 0.271 & 0.093 & 0.124 & 0.0 & 0.0 \\
\hline Reflector & 1 & 1.41 & 0.0 & 0.0 & 0.0 & 0.0476 \\
& 2 & 0.117 & 0.0191 & 0.0 & 0.0 & 0.0 \\
\hline
\end{tabular}




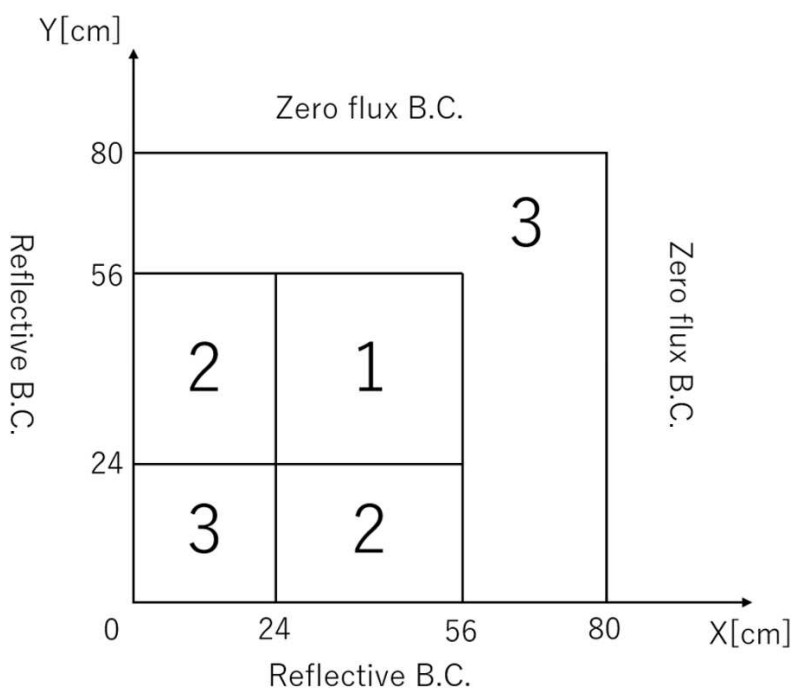

Figure 1: TWIGL core geometrical specification 


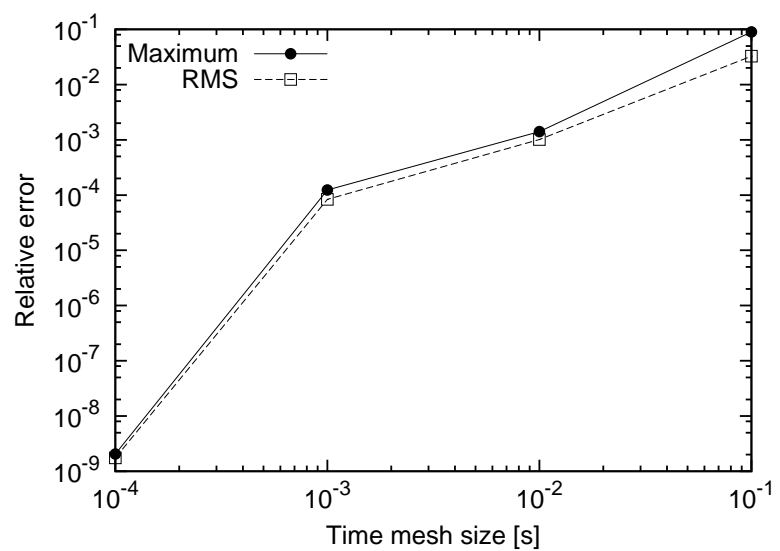

Figure 2: Maximum and RMS errors of the PME method with different time mesh sizes for reactor power 

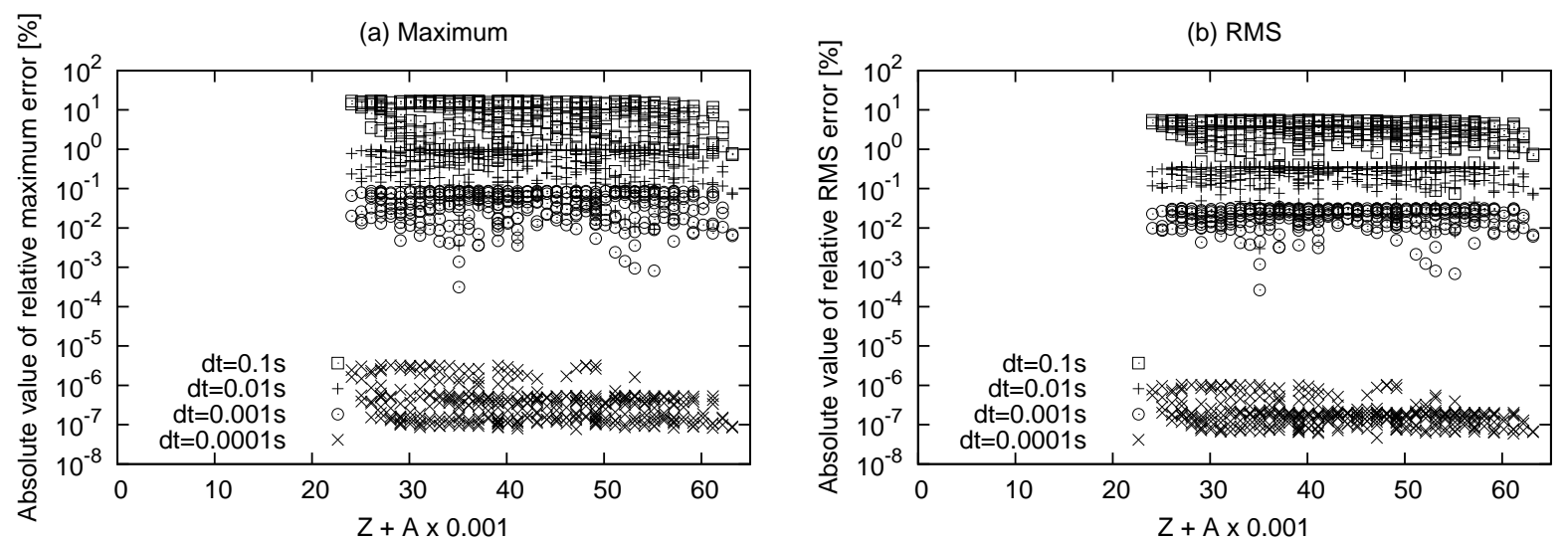

Figure 3: Maximum and RMS errors of the PME method with different time mesh sizes for FP number densities. $Z$ and $A$ are atomic number and mass number, respectively. 
(a) Power

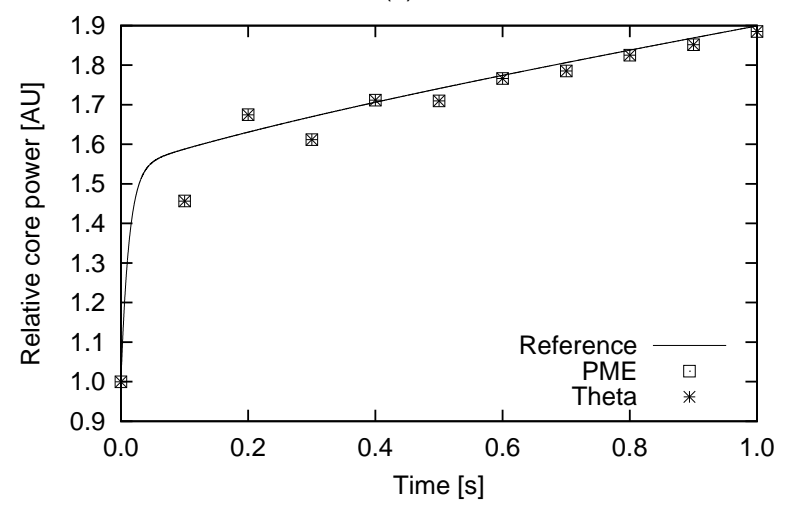

(b) Co-78 number density

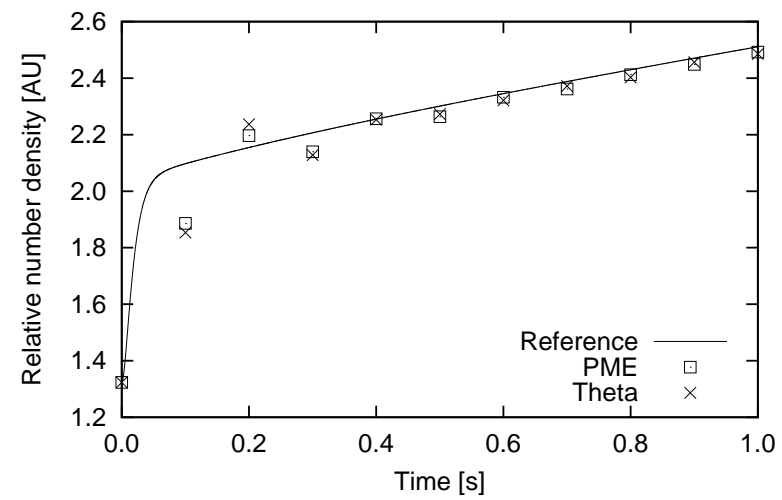

Figure 4: Reactor power and Co-78 number density obtained by the PME method and the $\theta$ method with $\Delta t=0.1 \mathrm{~s}$ 

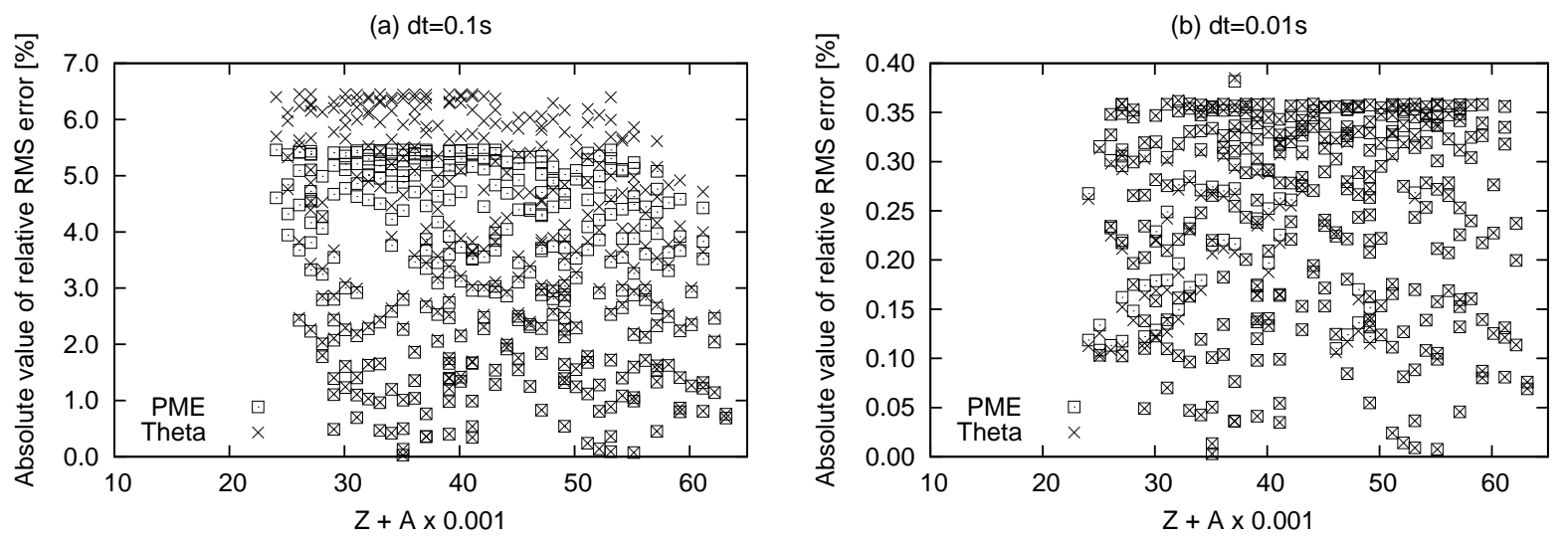

Figure 5: RMS errors of FP number densities of the PME method and the $\theta$ method 


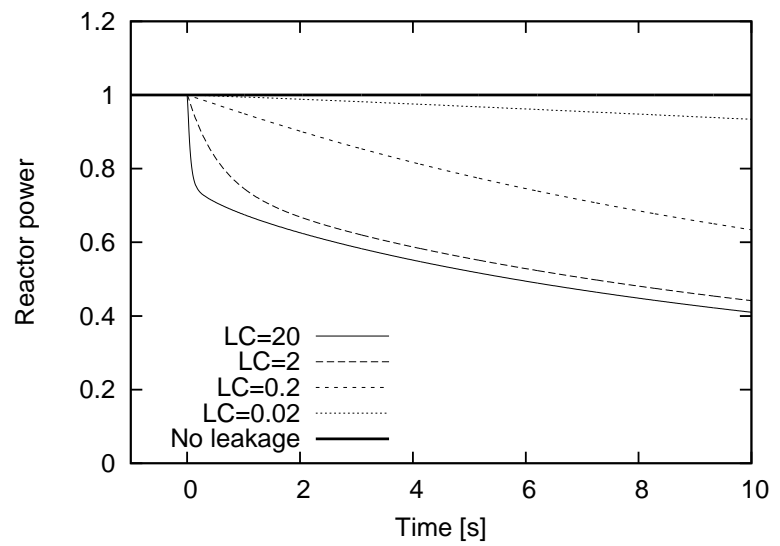

Figure 6: Reactor power change in a case of gaseous FP leakage in a critical system 

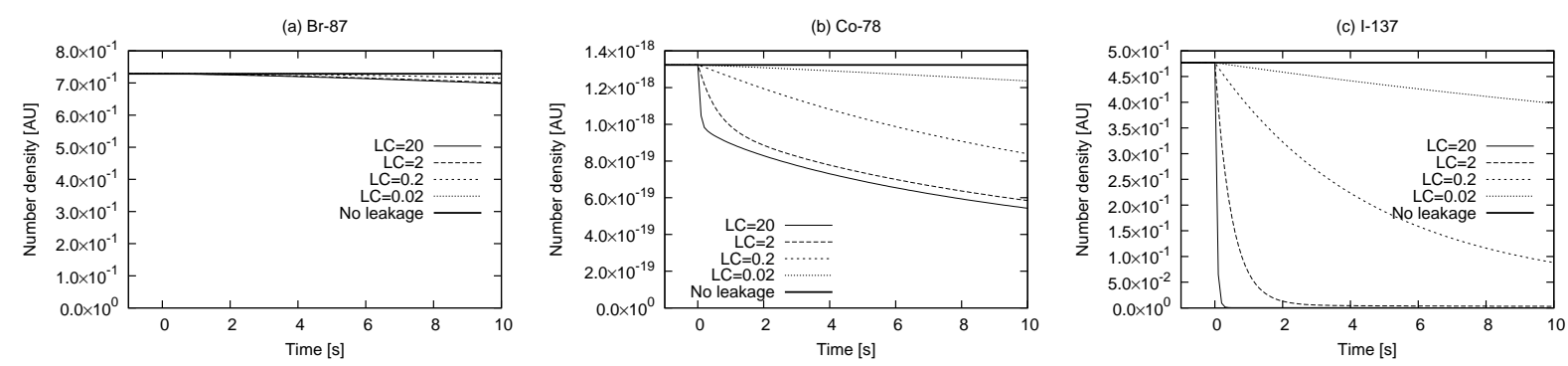

Figure 7: Number densities change in a case of gaseous FP leakage in a critical system 


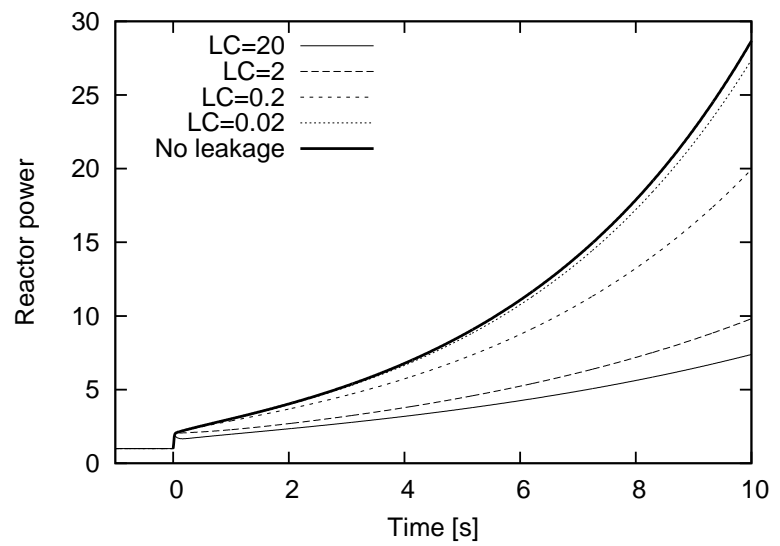

Figure 8: Reactor power change in a case of gaseous FP leakage with step-wise positive reactivity insertion 

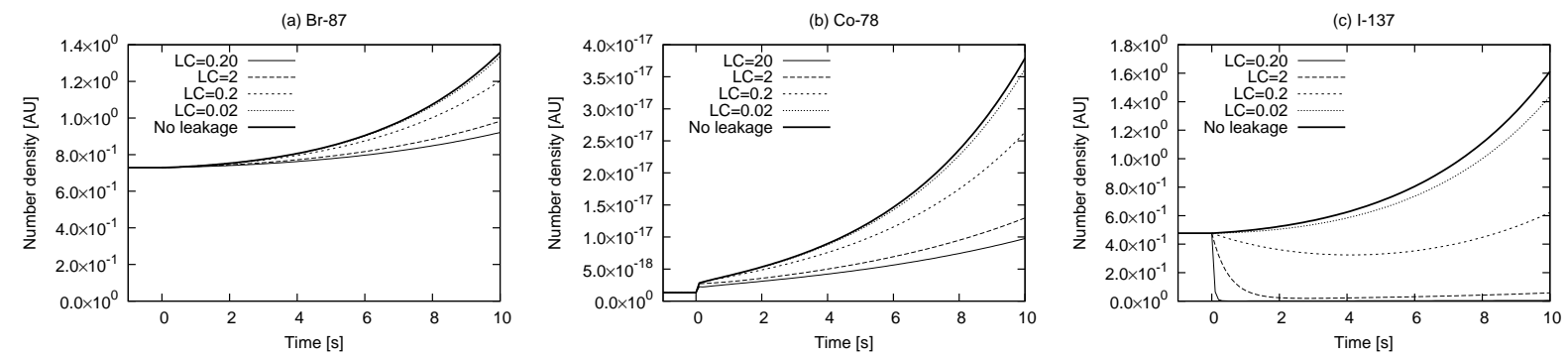

Figure 9: Number densities change in a case of gaseous FP leakage with step-wise positive reactivity insertion 


\section{List of Figure Captions}

Fig.1 TWIGL core geometrical specification

Fig.2 Maximum and RMS errors of the PME method with different time mesh sizes for reactor power

Fig.3 Maximum and RMS errors of the PME method with different time mesh sizes for FP number densities. $Z$ and $A$ are atomic number and mass number, respectively.

Fig.4 Reactor power and Co-78 number density obtained by the PME method and the $\theta$ method with $\Delta t=0.1 \mathrm{~s}$

Fig.5 RMS errors of FP number densities of the PME method and the $\theta$ method

Fig.6 Reactor power change in a case of gaseous FP leakage in a critical system

Fig.7 Number densities change in a case of gaseous FP leakage in a critical system

Fig.8 Reactor power change in a case of gaseous FP leakage with step-wise positive reactivity insertion

Fig.9 Number densities change in a case of gaseous FP leakage with step-wise positive reactivity insertion 